\title{
Effect of Self-administered Transcranial Direct Stimulation in Patients with Major Depressive Disorder: A Randomized, Single-blinded Clinical Trial
}

\author{
Jihoon Oh, Kuk-In Jang, Sekye Jeon, Jeong-Ho Chae \\ Department of Psychiatry, Seoul St. Mary's Hospital, College of Medicine, The Catholic University of Korea, Seoul, Korea
}

Objective: In numerous studies that have addressed transcranial direct current stimulation (tDCS) devices, participants visit the hospital regularly and undergo stimulation directed by health professionals. This method has the advantage of being able to deliver accurate stimuli in a controlled environment, but it does not adopt the merits of tDCS portability and applicability. Thus, it may be necessary to investigate how self-administered tDCS treatment at home affects depression-related symptoms.

Methods: In this randomized, single-blinded clinical trial, 58 patients with major depressive disorder were assigned to active and sham tDCS stimulation groups, and treatment responses were evaluated biweekly over six weeks. Both active and sham tDCS treatment group were treated with escitalopram. All participants were instructed the protocol and usage of at-home tDCS device, and self-administered tDCS treatment at their home.

Results: The beck-depression inventory score decreased significantly as treatment progressed, and the degree of symptom improvement was significantly higher in the active group than in the sham tDCS group. There were no significant differences between the two groups in other indices, including the Hamilton Depression Scale.

Conclusion: These results suggest that patient-administered tDCS treatment might be effective in improving subjective symptoms of depression.

KEY WORDS: Transcranial direct current stimulation; Depression; Therapuetics; Home-settings.

\section{INTRODUCTION}

Major depressive disorder (MDD) is a severe psychiatric illness that causes various psychological and cognitive symptoms that eventually lead to deterioration of daily functions [1]. Patients with MDD share similar psychiatric symptoms, but their progress and responses to treatment are highly variable, complicating therapeutic management of depression [2].

Cross-country evidence suggests that the prevalence of depression is consistent with sociodemographic correlates [3]. In South Korea, the lifetime prevalence of major

Received: October 25, 2020 / Revised: December 27, 2020 Accepted: December 31, 2020

Address for correspondence: Jeong-Ho Chae

Department of Psychiatry, Seoul St. Mary's Hospital, College of Medicine, The Catholic University of Korea, 222 Banpo-daero, Seocho-gu, Seoul 06591, Korea

E-mail: alberto@catholic.ac.kr

ORCID: https://orcid.org/0000-0002-6070-9324 depressive disorder was $5.0 \%(3.0 \%$ in males and $6.9 \%$ in females) among the entire population, and the annual prevalence rate was $1.5 \%$ (1.1\% in males and $2.0 \%$ in females) in 2016 [4]. However, the number of patients who actually visit the hospital and receive treatment is much smaller than that of patients suffer from MDD [5].

Although various treatment methods have been introduced including antidepressants, psychotherapy, electroconvulsive therapy, and the combination of these therapeutics [6], due to the heterogenicity of the illness, the response to treatment is widely inconsistent [2]. About two-thirds of patients in a previous study diagnosed with MDD were receiving related treatments, and only one-third of patients were taking antidepressant medications, which is the first-line therapy option for managing MDD [7]. This relatively low rate of antidepressant usage is also correlated with patient reluctance to initiate medication due to worries of side effects and drug addiction [8] and only 25 to 50 percent of depressive patients adhere to the anti-

(c) This is an Open-Access article distributed under the terms of the Creative Commons Attribution Non-Commercial License (http://creativecommons.org/licenses/by-nc/4.0) which permits unrestricted non-commercial use, distribution, and reproduction in any medium, provided the original work is properly cited. 
depressant treatment [9]. Thus, a number of non-pharmacological treatments (NPT) have been developed and the concurrent treatment of NPTs was related to higher adherence to the treatment for depression [10]. Furthermore, the combined usage of antidepressant and neurostimulation methods showed significant effects on the treatment of depression in the case of transcranial magnetic stimulation [11] and electro-convulsive therapy [12]

Recently, transcranial direct current stimulation (tDCS), which sends low and constant direct current through electrodes to the dorsolateral prefrontal area, has been widely examined, considering its potential to improve depression-related symptoms [13-16]. A meta-analysis of patients with acute major depressive episodes revealed that active tDCS treatment was significantly effective than the sham condition and the effect size was comparable to that of classical transcranial magnetic stimulation (TMS) therapy [14]. A large-scale randomized control study that investigated the effects of antidepressants and tDCS on patients with unipolar depression, tDCS treatment was not inferior to antidepressant medication (e.g., escitalopram) but had more side effects [11].

Although tDCS devices have the advantage of being safe and more portable than TMS or electroconvulsive therapy, most previous studies were conducted in circumstances in which patients visited a hospital and were treated by health professionals. However, as patients receiving depression treatment may experience an inability to visit the hospital regularly, it is necessary to investigate whether known treatment effects of tDCS could be maintained at patients' homes. Therefore, this study sought to confirm the effectiveness of self-application of a portable tDCS device that could be used at home and managed through a mobile application.

\section{METHODS}

\section{Trial Design}

In this randomized, single-blinded clinical trial, 58 patients with major depressive disorder without psychotic features were assigned to active and sham tDCS stimulation groups in a 1:1 ratio, and treatment responses were evaluated biweekly over six weeks. Enrollment, allocation and follow-up procedures are presented in Figure 1. Twenty nine were allocated in active group and a total of 9 participants were discontinued during the interven-

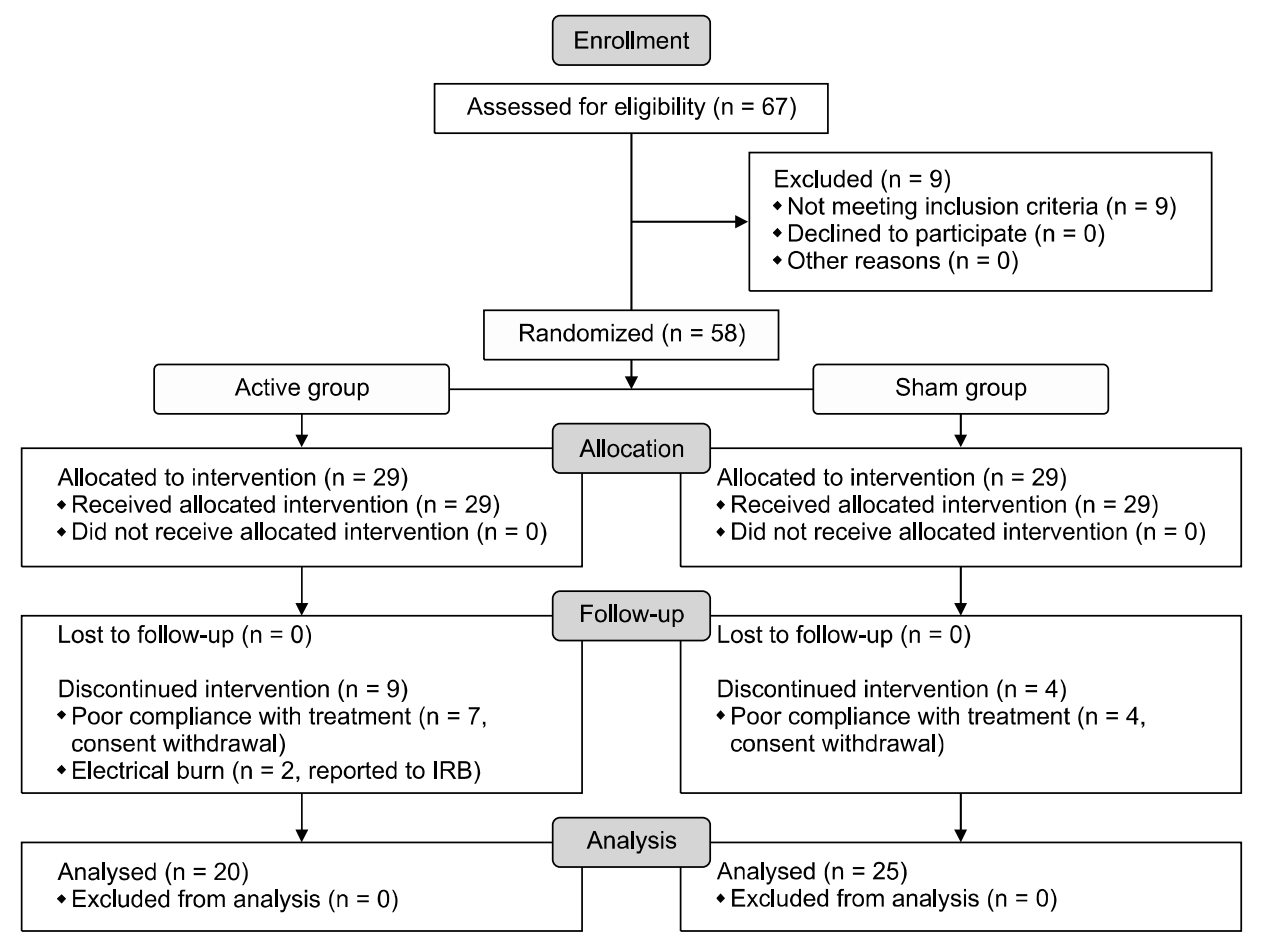

Fig. 1. Enrollment, allocation of groups and follow-up processes are presented with CONSORT flow-diagram. CONSORT, consolidated standards of reporting trials; IRB, institutional review board. 


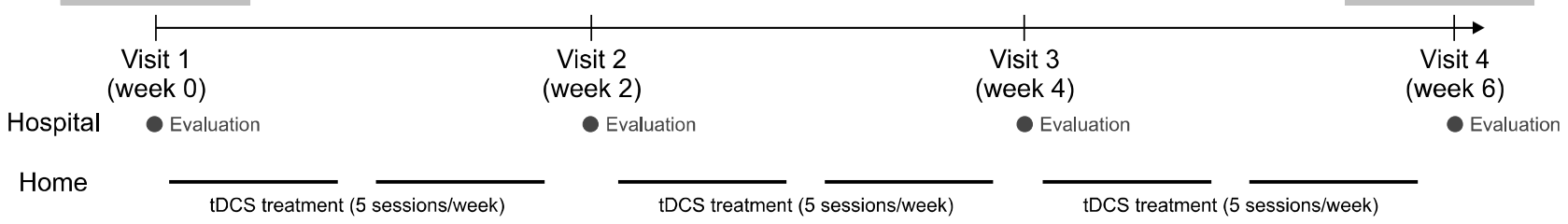

Fig. 2. Study design and participant selection. tDCS, transcranial direct current stimulation.

tion. Two of them had a mild electrical burn on their forehead and 7 had a poor compliance with treatment. Among sham group ( $n=29$ ), a total of 4 participants halted the intervention due to their poor compliance to the experiment. The procedure and timeline of the trial are shown in Figure 2. Participants had visited the hospital every two weeks and the raters who measured clinical-rated scales did not notice whether the participants were belonged to active or sham groups.

\section{Participants and Case Definition}

Among inpatients and outpatients who were admitted to or visited Seoul St. Mary's Hospital, The Catholic University of Korea, adults aged 19 to 65 years with diagnosis of MDD (based on the criteria of Diagnostic and Statistical Manual of Mental Disorders, 5th edition) were enrolled in this study. Participants with a history of alcohol or substance abuse and dependence, severe brain disease and trauma, neurological and motor disorders, at risk for suicide, who were pregnant, or who were showing mental disorders other than MDD and anxiety disorder were excluded (among anxiety disorders, patients with post-traumatic stress disorder and obsessive-compulsive disorders were not allowed to participate in the study). Participants with a total score of 22 points or higher on the Montgomery-Åsberg depression rating scale (MADRS) who had moderate to severe depressive symptoms were enrolled in the study [17].

At baseline, a total of 67 participants was screened, and 58 were enrolled in the randomization process. As a result, 58 participants were equally split between the active and sham tDCS groups (antidepressant medications + active $t D C S=29$ patients and antidepressant medication + sham $\mathrm{tDCS}=29$ patients). There were 13 dropouts during the six-week period of tDCS treatment, including nine from the active tDCS group and four from the sham tDCS groups (Fig. 1). Thus, 20 participants in the active tDCS group and 25 participants in the sham tDCS group finalized the six-week treatment protocol, and the data of these participants were analyzed in this study.

All subjects who participated in this study provided written informed consent, and the study was approved by the Institutional Review Board of the Ethics Committee of Seoul St. Mary's Hospital at The Catholic University of Korea (KC09FZZZ0211).

\section{Usage of Psychiatric Medication}

Participants in both active and sham groups were prescribed escitalopram $5-20 \mathrm{mg} /$ day as conventional treatment in combination with tDCS treatment. Patients who were previously given antidepressants, antipsychotics, or anticonvulsants require a washout period of at least five drug half-lives (e.g., venlafaxine hydrochloride and paroxetine hydrochloride for at least two weeks, fluoxetine hydrochloride for at least five weeks). Concomitant usage of anxiety drugs was allowed; in this context, diazepam could be used up to a maximum dose of $20 \mathrm{mg}$ per day, while benzodiazepine drugs other than diazepam were allowed at a dose equivalent to diazepam $20 \mathrm{mg}$ per day.

\section{Protocol of tDCS Treatment}

A randomized, single-blinded clinical trial was conducted for six weeks. Participants visited the hospital four times (biweekly for two weeks) (Fig. 2). During their second visit, a research nurse instructed each participant on use of home-based tDCS equipment (YDS-301N; Ybrain Inc., Seongnam, Korea), and all participants thereafter conducted tDCS treatment at home once a day for $30 \mathrm{mi}$ nutes (five times per week). On the day on which participants visited a hospital (baseline and weeks 2, 4, and 6), 
a research nurse applied the stimulation with the same type of tDCS equipment (30 minutes/session for one session), and the participant was told not to use the device again that day. Participants were instructed to install application that control portable tDCS device. This application allows us to check whether the device was properly used and specific parameters were recorded to verify the compliance of treatment (duration of stimulation, interval of each session, mean current intensity etc.). To enhance the compliance of the home-based tDCS treatment, participants could access online manuals (https://youtu.be/ 19kNeUPLmZA, https://youtu.be/CBJPBiRWgXQ) before they use tDCS device at home.

In the active tDCS treatment group, $2 \mathrm{~mA}$ of current was delivered during the 30 minutes of treatment. In the sham tDCS treatment group, the current rose slowly for 30 seconds, descended for 30 seconds, and then remained at zero for 29 minutes. The anode was positioned at the left dorsolateral frontal cortex, and the cathode was positioned at the right lateral frontal cortex. The dorsolateral prefrontal cortex corresponds to Brodmann regions 9 and 46 and corresponds to the F3 and F4 electrode regions of the electroencephalography 10-20 system. Thus, location of the anode was similar to that of $\mathrm{F} 3$, while the cathode was attached at F4. Dosage and types of psychiatric medications remained unchanged. The protocol of this study was registered in clinicalstrial.gov (ClinicalTrials.gov ID: NCT04543123).

\section{Outcome Measurements}

The primary outcome of this study was improvement of a total score of objective and self-rated scales for depression. The Hamilton depression scale (HAM-D) [18] was deployed by a trained psychiatrist at every visit (weeks 0, 2, 4, and 6) and was used to measure the degree of depression observed by clinician. Observer-rated MADRS [19] which is known to more sensitive to the changes of depressive symptoms caused by treatment was also addressed [20]. To measure the subjective complaints of depression, self-rated scale of the beck depression inventory (BDI) [21] and Perceived Deficits Questionnaire-Depression (PDQ-D) $[22,23]$ were completed by participants.

We also administered 8 other psychiatric scales, Hamilton anxiety scale [24], beck anxiety inventory [25], statetrait anxiety inventory (S-TAI) [26], Cognitive Emotion
Regulation Questionnaire [27], Connor-Davidson resilience scale [28], and mini-mental status examination (MMSE) [29]. Basic demographic features including sex and age were also evaluated.

\section{Sample Size}

In the results of the most similar clinical study that examined the effect of tDCS on cognitive function in the past [30], there was a difference in MMSE value of 2.2 in the test group treated with tDCS compared to the control group, and the standard deviation estimate is, 3.0, the standard deviation of the indicator, was used after tDCS. Based on this, the number of samples for comparison of the mean (superiority) of independent samples is calculated. Calculated by the two-sided test with significance level $\alpha=$ 0.05 and power $90 \%$ (1-B).

$$
\mathrm{n}=\frac{2 \sigma^{2}\left(\mathrm{z}_{\alpha / 2}+\mathrm{z}_{\beta}\right)^{2}}{\mathrm{~d}^{2}} \cong 39.06
$$

Based on the above formula, the number of samples in one group considering the drop-out rate $(7.5 \%)$ is 42 , and since it was a two-group test, the total number of samples was calculated as 84 .

\section{Randomization and Blinding}

This clinical trial was a single blind clinical trial, and only those who meet the criteria for inclusion/exclusion in the baseline were given an enrollment number from the investigator. The order of medical device allocation according to the subject enrollment number was programmed using the latest version of SAS statistical software version 9.4 (SAS Institute, Inc., Cary, NC, USA) by the block randomization method in consideration of the block size specified in advance by the statistician of this clinical trial. Through randomization, the study group/control group assignment of each subject enrollment number was determined with corresponding active tDCS and sham tDCS equipment which had the same appearance, display, and sound indication. For blinding in the sham tDCS treatment group, the stimulation current rose slowly for 30 seconds, descended for 30 seconds, and then remained at zero for 29 minutes.

\section{Statistical Analysis}

Independent $t$ test was used in discriminating between 
A

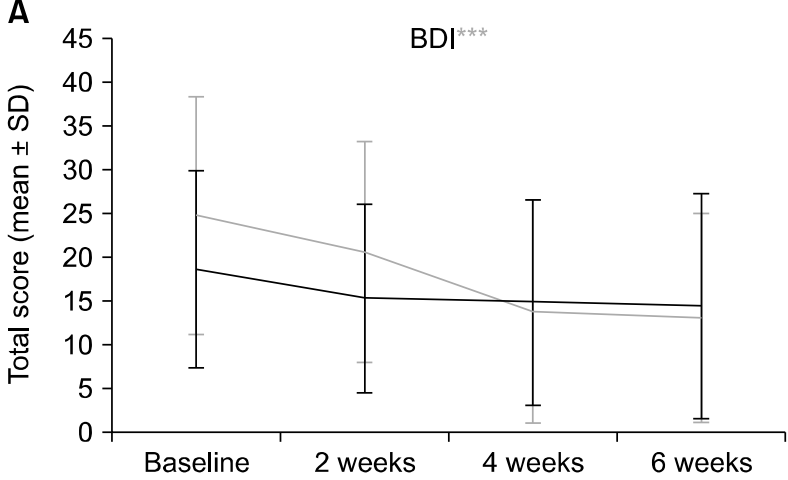

C

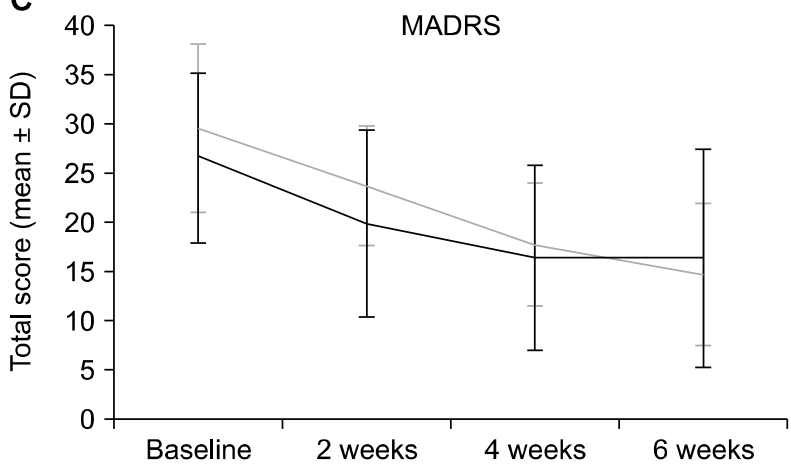

B

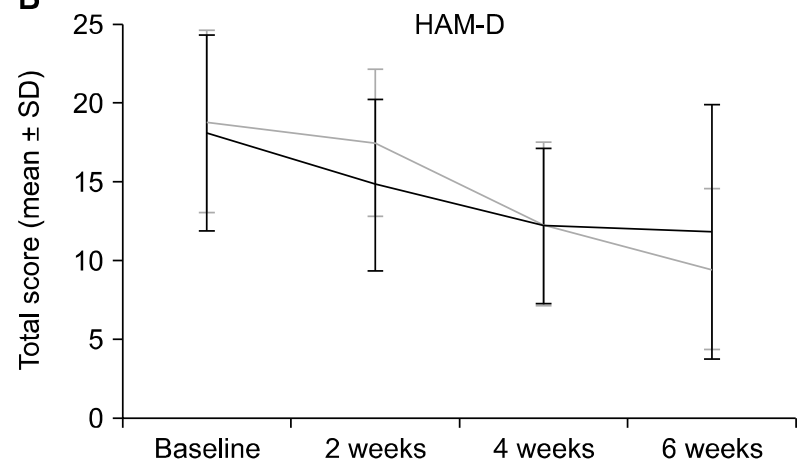

D

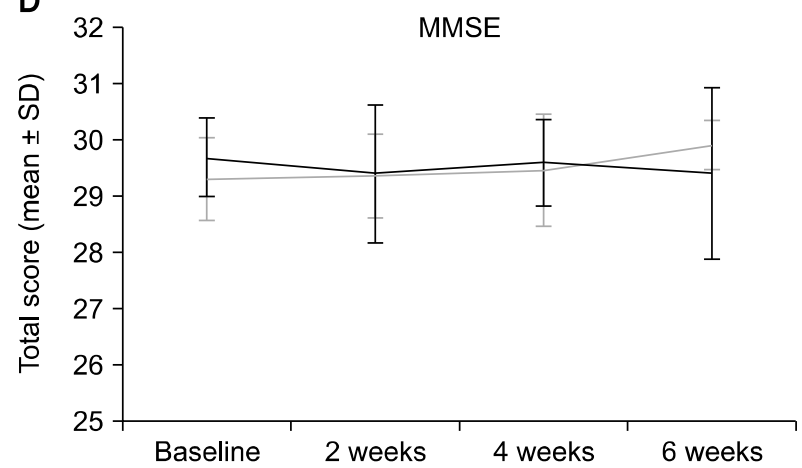

Fig. 3. Total score changes on psychiatric scales during the six-week treatment period: (A) beck-depression inventory (BDI), (B) Hamilton depression scale (HAM-D), (C) Montgomery-Åsberg depression rating scale (MADRS), and (D) mini-mental status examination (MMSE). Error bar denotes standard deviation (SD) in each point.

$* * * p<0.001$.

demographic and baseline characteristics of active and sham tDCS treatment groups. Repeated-measures analysis of variance was administered to evaluate the timevarying covariates of each outcome measurement between the active and sham tDCS groups. General linear model with dependent variables of each psychiatric scale's total score at the time of measurement (baseline, 2 weeks, 4 weeks and 6 weeks) was constructed and multivariate test with time and time*group effect was analyzed. Test of within-subjects effects was measured with a sphericity assumed hypothesis and the results of within-subjects contrasts was used to describe the time and time*group effect of tDCS treatment. Tests of between subjects effects was used to measure the group effect on the treatment of tDCS. Chi-square analysis was done to evaluate statistical differences between active and sham groups in following variables (i.e., education level, hospitalization, a number of responders and remitters). All statistical analyses were performed using Statistical Package for the Social Sciences version 23.0 (IBM Corp., Armonk, NY, USA).

\section{RESULTS}

\section{Demographic and Baseline Psychiatric Scales of the Active and Sham Groups}

Table 1 shows the results of demographic information and psychiatric scales of the active $(\mathrm{n}=20)$ and sham $(\mathrm{n}=$ 25) stimulation groups. There was no difference in age between the two groups, but the percentage of women was significantly higher in the active group than in the sham group (50\% vs. $40 \%$; $p<0.001)$.

Twelve psychiatric scales including HAM-D and BDI were used to identify the baseline psychiatric status of the active and sham stimulation groups, and no significant differences were found except in the S-TAI (active group vs. sham group, mean \pm standard deviation [mean $\pm \mathrm{SD}$ ]: $65.6 \pm 9.6$ vs. $58.8 \pm 9.8, p=0.03)$. Although the subjective level of anxiety was higher in the active stimulation group, outcomes according to the Hamilton Anxiety Scale deployed by the psychiatrist were not significantly different between the groups (active group vs. 
Table 1. Demographic and baseline characteristics of active and sham groups

\begin{tabular}{lccc}
\hline \multicolumn{1}{c}{ Variable } & Active group $(\mathrm{n}=20)$ & Sham group $(\mathrm{n}=25)$ & $p$ value \\
\hline Sex, female (\%) & 50.0 & 40.0 & 0.001 \\
Age (yr) & $29.7 \pm 11.6$ & $28.5 \pm 11.0$ & 0.72 \\
Education level & & & $0.69^{\mathrm{a}}$ \\
$\quad$ Bachelor's degree, yes (\%) & 75.0 & 80.0 & 0.45 \\
Duration of illness (mo) & $32.3 \pm 32.6$ & $25.1 \pm 30.9$ & \\
Comorbid psychiatric illness & & & \\
Anxiety disorder, yes (\%) & 25.0 & 16.0 & 0.89 \\
Escitalopram dosage (during the study, mg) & $16.3 \pm 6.5$ & $16.0 \pm 6.3$ & 0.66 \\
Escitalopram dosage (before the study, mg) & $(\mathrm{n}=8) 13.0 \pm 5.9$ & $(\mathrm{n}=8) 12.0 \pm 5.3$ & $0.37^{\mathrm{a}}$ \\
Psychiatric Inpatient care, yes (\%) & 0.0 & 4.0 & 0.26 \\
Total score of psychiatric scales & & & 0.08 \\
MADRS & $29.5 \pm 8.5$ & $26.6 \pm 8.6$ & 0.69 \\
MMSE & $29.3 \pm 0.7$ & $29.7 \pm 0.7$ & 0.17 \\
HAM-D & $18.8 \pm 5.8$ & $18.1 \pm 6.2$ & 0.61 \\
HAM-A & $21.7 \pm 8.4$ & $18.1 \pm 8.5$ & 0.12 \\
PDQ-D & $31.4 \pm 16.5$ & $28.7 \pm 18.9$ & 0.14 \\
BDI & $27.0 \pm 12.0$ & $21.6 \pm 10.0$ & 0.05 \\
BAl & $22.7 \pm 15.7$ & $16.5 \pm 10.4$ & 0.03 \\
SAl & $61.0 \pm 11.0$ & $54.6 \pm 9.4$ & 0.25 \\
TAl & $65.6 \pm 9.6$ & $58.8 \pm 9.8$ & 0.44 \\
CERQ & $98.4 \pm 15.3$ & $93.0 \pm 15.3$ & 0.88 \\
CDRS & $33.2 \pm 15.5$ & $36.9 \pm 15.8$ & \\
SDS & $18.0 \pm 7.8$ & $17.6 \pm 7.2$ & \\
\hline
\end{tabular}

Values are presented as mean \pm standard deviation.

MADRS, Montgomery - ̊̊sberg depression rating scale; MMSE, mini mental status examination; HAM-D, hamilton depression scale; HAM-A, Hamilton anxiety scale; PDQ-D, perceived deficits questionnaire-depression; BDI, beck depression inventory; BAI, beck anxiety inventory; SAI, state anxiety inventory; TAI, trait anxiety inventory; CERQ, cognitive emotion regulation questionnaire; CDRS, Connor-Davidson resilience scale; SDS, self-rating depression scale.

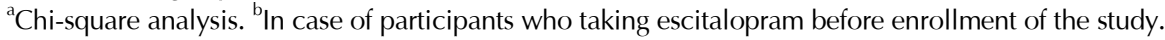

sham group: $21.7 \pm 8.4$ vs. $18.1 \pm 8.5 ; p=0.17)$.

\section{Effect of tDCS on Improvement of Depressive Symptoms}

Figure 3 shows the results of temporal changes of two indicators of depression (HAM-D and BDI) during tDCS treatment. BDI score, indicating subjective depressive symptoms, significantly improved in both the active and sham tDCS groups as treatment progressed (Fig. 3A), although the increase in BDI score was significantly different between the active and sham tDCS groups; time* group effect of BDI score was greater in active group than sham tDCS groups (time effect: $\mathrm{F}=41.76, \mathrm{df}=1, p<0.001$; time*group effect: $\mathrm{F}=11.29, \mathrm{df}=1, p<0.01$; group: $\mathrm{F}=$ 0.43, $\mathrm{df}=1, p=0.52$ ).

Total HAM-D score was also improved in both the active and sham tDCS groups as treatment progressed, but no significant difference was found between these two groups (Fig. 3B, time effect: $\mathrm{F}=59.87, \mathrm{df}=1, p<0.001$; time* group effect: $\mathrm{F}=2.81, \mathrm{df}=1, p=0.10$; group: $\mathrm{F}=$ $0.04, \mathrm{df}=1, p=0.85)$. Similar to the two indicators above, a total MADRS score significantly decreased as number of treatment sessions increased, though we could not discern any statistical differences between active and sham tDCS groups (Fig. 3C, time effect: $\mathrm{F}=63.43, \mathrm{df}=1, p<$ 0.001 ; time* group effect: $\mathrm{F}=2.37, \mathrm{df}=1, p=0.13$; group: $\mathrm{F}=0.61, \mathrm{df}=1, p<0.44)$. The change of PDQ-D total score was also similar to other psychiatric scales (time effect: $\mathrm{F}=7.25, \mathrm{df}=1, p=0.10$ : time* group effect: $\mathrm{F}=1.12$, $\mathrm{df}=1, p=0.29$; group: $\mathrm{F}=0.02, \mathrm{df}=1, p=0.90)$.

Since the active tDCS group showed a larger BDI score change relative to the sham tDCS group, we further analyzed the change in BDI score before and after treatments (Fig. 4). The decrement in BDI score before and after treatment was significantly greater in the active tDCS group than in the sham tDCS group (active group vs. sham group: mean $\pm \mathrm{SD}, 10.40 \pm 7.98$ vs. $3.80 \pm 7.01 ; \mathrm{t}=2.95$, $p<0.05)$. This outcome suggests that the degree of im- 
BDI

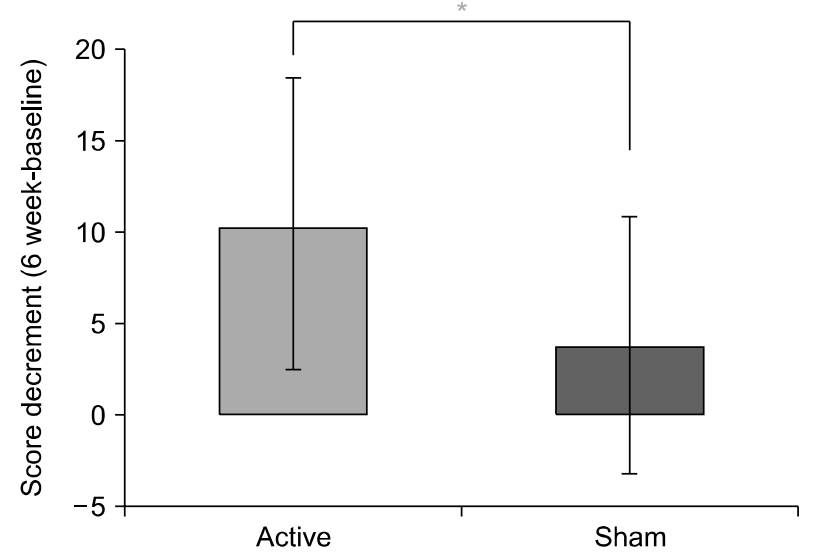

Fig. 4. Decrement of total BDI score between posttreatment and baseline in the active and sham tDCS treatment groups.

BDI, beck-depression inventory; tDCS, transcranial direct current stimulation.

${ }^{*} p<0.05$.

provement of subjective depression in the active tDCS group was higher than that in the sham tDCS group.

To confirm the improvement of degree of depression in the active and sham tDCS groups, we measured the ratio of responders ( $>50 \%$ decrease in MADRS scores) and remitters (MADRS $<10$ or HAM-D $<7$ at the endpoint) in both groups, but no statistical significance was found (a number of responders in active group vs. sham group, 10 vs. $9, p=0.34$; a number of remitters in active group vs. sham group, 7 vs. 10, $p=0.73$, chi-square analysis).

\section{Effect of tDCS Treatment on Cognition and Side Effects}

To investigate whether the six weeks of tDCS treatment affected short-term cognition, we measured the score of the MMSE at each visit. When comparing the data from the initial visit and six weeks after treatment, there was no significant change in MMSE score in either the active or sham tDCS groups and no significant difference in change in MMSE score between the two groups over time (Fig. 3). Among 45 participant, one reported mild headache after the tDCS treatment but soon resolved symptoms and completed the experiment. Two of 20 participants in the active group showed a mild electrical burn. They were referred to dermatology immediately and fully recovered after the treatment. Other side effects were not reported in these participants.

\section{DISCUSSION}

In this study, we showed that at-home usage of tDCS devices was effective in improving subjective feeling of depression, as represented by total BDI score. The degree of improvement among depression-related symptoms experienced by patients was significantly higher in the active tDCS stimulation group than in the sham group but without a significant difference when measured using total HAM-D. In addition, short-term tDCS treatment for six weeks had no significant effect on MMSE score.

Our results are comparable to previous important findings of the effect of tDCS on depression [31]. Loo et al. [32] showed that 15 sessions over three weeks of 2-mA tDCS treatment significantly improved mood symptoms. In this study, the primary outcome measurement of MADRS showed a significant interaction between group and time, but no significant differences were found between the sham and active groups. Another sham-controlled, double-blinded study that targeted treatment-resistant major depressive disorder patients found that scores on the Hamilton Rating Scale for Depression-17 scale were not significantly different between active and sham tDCS groups [33].

A recent randomized controlled tDCS trial also found that MADRS score significantly improved with continuing treatment, but no significant difference was found between active and sham groups [34]. Our results reveal similar outcomes to those of previous studies. With an increase in number of tDCS treatment sessions, scores associated with depression-related symptoms (i.e., HAM-D, BDI, and MADRSs) seemed to improve. However, only a total score of BDI was ultimately significantly different between the active and sham tDCS treatment groups. These results suggest that, in cases where patients use devices themselves at home, tDCS is effective in terms of improving subjective symptoms of depression, similar to when being treated by clinical experts at the hospital.

Our results of a significant difference in only BDI score between active and sham tDCS groups support that subjective symptoms of depression might be correlated with the characteristics of at-home tDCS treatment. Unlike in conventional tDCS administered by clinicians in the hospital, self-administered tDCS at the patient's home requires both continued efforts and motivation on their part. The willingness to continue treatment might be related to 
a substantial improvement of symptoms in the sham group and might contribute to the nonsignificant differences between the active and sham tDCS groups.

We also found that improvement in total BDI score was greater at the beginning of treatment than in later stages (Fig. 3). The decrement in BDI score was prominent in both active and sham groups until the second week of treatment, while degree of improvement slowed after the fourth week. Although it was not statistically significant, the degree of MADRS score improvement was also more pronounced in the first four weeks after treatment than between four and six weeks.

Our findings suggest a noticeable improvement of depressive symptoms immediately following initiation of tDCS treatment. A prior study that applied tDCS in patients with phantom limb pain reported an immediate and sustained effect on relief of neuropathic pain with five days of active treatment [35]. Other clinical tDCS trials on depression measured primary outcomes within two to four weeks after treatment (five times/week), and improvements in depression-related symptoms mainly occurred during this period [36]. Our findings suggest that the effect of tDCS appears predominantly after initiation of treatment.

Although quantitative aspects of cognition were not used, we found that active stimulation of tDCS treatment did not significantly affect cognitive function, as measured by total MMSE score. It has been reported that tDCS treatment has no reliable effect on various dimensions of cognitive function (e.g., executive function, language, memory) in the healthy population [37] and showed no cognitive benefits in MDD patients [38]. Considering these results, our findings suggest that tDCS treatment within six weeks may not result in short-term cognitive decline or improvement.

Our results should be interpreted in their context with several limitations. First, participants in both active and sham tDCS groups were prescribed escitalopram during the enrollment period of the study. Thus, the nonsignificant differences in scores of depression-related scales except BDI between groups might be the result of treatment effects of antidepressants. Second, although we found that improvement in total BDI score was more prominent in the active group, that of the total HAM-D score was not. Therefore, there remains a possibility that an improvement in depression symptoms could fall within the sub- threshold range in which clinicians cannot detect. Third, as three different psychiatric scales were used to assess depressive symptoms (MADRS, HAM-D, and BDI), there is a possibility of the type II errors in our results. Fourth, there was a statistically significant difference in sex ratio between the groups. Although a recent study suggested that sex might be not a predictor of treatment response [39], findings in this study could be affected by the larger number of females in the active tDCS group. Fifth, we inherently could not compare the effect of tDCS with the effect of antidepressants. Further investigation is needed to compare the effects of at-home tDCS treatment - only and antidepressant-only groups. Sixth, the enrolled patient in this study was less than initially planned (20 of 29 in active, 25 of 29 in sham groups) and they were excluded in the analysis. As a commonly used method of imputing data with dropouts, such as the last observation carried forward was not used, the results reported in this study may lead to bias.

In conclusion, our findings suggest that self-administered tDCS with an antidepressant treatment among MDD patients may have a significant effect on the improvement of total BDI score. Other measurements of depression, such as HAM-D and MADRS scores, were not significantly different between active and sham tDCS groups. Treatment response was usually noticeable at two to four weeks after start of treatment, and there was no significant change in MMSE score in either the active or sham tDCS group. This study suggests that self-administering tDCS treatment at home could be effective in improving the subjective symptoms of depression.

\section{- Funding}

This research was supported by a grant from the Korea Health Technology R\&D Project through the Korea Health Industry Development Institute (KHIDI), funded by the Ministry of Health \& Welfare of the Republic of Korea (grant no. HL19C0007 and HI17C2272).

\section{- Conflicts of Interest}

No potential conflict of interest relevant to this article was reported.

\section{Author Contributions}

Concept and design of study: Jeong-Ho Chae. Acquisition of data: Sekye Jeon, Kuk-In Jang. Analysis and inter- 
pretation of data: Jihoon Oh, Kuk-In Jang, Jeong-Ho Chae. Drafting the manuscript: Jihoon Oh. Revising the manuscript critically for important intellectual content: Kuk-In Jang, Jeong-Ho Chae.

\section{ORCID}

Jihoon $\mathrm{Oh}$

https://orcid.org/0000-0003-1114-976X

Kuk-In Jang https://orcid.org/0000-0002-7405-3899

Sekye Jeon https://orcid.org/0000-0002-8937-6025

Jeong-Ho Chae

https://orcid.org/0000-0002-6070-9324

\section{REFERENCES}

1. Dufouil C, Fuhrer R, Dartigues JF, Alpérovitch A. Longitudinal analysis of the association between depressive symptomatology and cognitive deterioration. Am J Epidemiol 1996;144: 634-641.

2. Belmaker RH, Agam G. Major depressive disorder. N Eng/J Med 2008;358:55-68.

3. Kessler RC, Bromet EJ. The epidemiology of depression across cultures. Annu Rev Public Health 2013;34:119-138.

4. Samsung Medical Center. The survey of mental disorders in Korea. Sejong:Ministry of Health and Welfare;2016.

5. Marcus SC, Olfson M. National trends in the treatment for depression from 1998 to 2007. Arch Gen Psychiatry 2010;67: 1265-1273.

6. Davidson JR. Major depressive disorder treatment guidelines in America and Europe. J Clin Psychiatry 2010;71 Supp/ E1:e04.

7. Ohayon MM. Epidemiology of depression and its treatment in the general population. J Psychiatr Res 2007;41:207-213.

8. Givens JL, Datto CJ, Ruckdeschel K, Knott K, Zubritsky C, Oslin DW, et al. Older patients' aversion to antidepressants. A qualitative study. J Gen Intern Med 2006;21:146-151.

9. Trivedi $\mathrm{MH}$, Lin EH, Katon WJ. Consensus recommendations for improving adherence, self-management, and outcomes in patients with depression. CNS Spectr 2007;12 (8 Supp/ 13): 1-27.

10. Park JH, Kim KW. A review of the epidemiology of depression in Korea. J Korean Med Assoc 2011;54:362-369.

11. Roman A, Vetulani J, Nalepa I. Effect of combined treatment with paroxetine and transcranial magnetic stimulation (TMS) on the mitogen-induced proliferative response of rat lymphocytes. Pol J Pharmacol 2002;54:633-639.

12. Gonzalez-Pinto A, Gutierrez M, Gonzalez N, Elizagarate E, Perez de Heredia JL, Mico JA. Efficacy and safety of venlafaxine-ECT combination in treatment-resistant depression. J Neuropsychiatry Clin Neurosci 2002;14:206-209.

13. Brunoni AR, Moffa AH, Sampaio-Junior B, Borrione L, Moreno $\mathrm{ML}$, Fernandes RA, et al. Trial of electrical direct-current therapy versus escitalopram for depression. N Engl I Med 2017; 376:2523-2533.
14. Brunoni AR, Ferrucci R, Fregni F, Boggio PS, Priori A. Transcranial direct current stimulation for the treatment of major depressive disorder: a summary of preclinical, clinical and translational findings. Prog Neuropsychopharmacol Biol Psychiatry 2012;39:9-16.

15. Shiozawa P, Fregni F, Benseñor IM, Lotufo PA, Berlim MT, Daskalakis JZ, et al. Transcranial direct current stimulation for major depression: an updated systematic review and metaanalysis. Int J Neuropsychopharmacol 2014;17:1443-1452.

16. Brunoni AR, Moffa AH, Fregni F, Palm U, Padberg F, Blumberger DM, et al. Transcranial direct current stimulation for acute major depressive episodes: meta-analysis of individual patient data. Br J Psychiatry 2016;208:522-531.

17. Bech P, Andersen HF, Wade A. Effective dose of escitalopram in moderate versus severe DSM-IV major depression. Pharmacopsychiatry 2006;39:128-134.

18. Williams JB. A structured interview guide for the Hamilton Depression Rating Scale. Arch Gen Psychiatry 1988;45:742747.

19. Svanborg P, Asberg M. A comparison between the Beck Depression Inventory (BDI) and the self-rating version of the Montgomery Asberg Depression Rating Scale (MADRS). J Affect Disord 2001;64:203-216.

20. Montgomery SA, Asberg M. A new depression scale designed to be sensitive to change. Br J Psychiatry 1979;134:382-389.

21. Beck AT, Steer RA, Carbin MG. Psychometric properties of the Beck Depression Inventory: twenty-five years of evaluation. Clin Psychol Rev 1988;8:77-100.

22. Lam RW, Saragoussi D, Danchenko N, Rive B, Lamy FX, Brevig T. Psychometric validation of Perceived Deficits Questionnaire-Depression (PDQ-D) in patients with major depressive disorder (MDD). Value Health 2013;16:A330.

23. Kim JM, Hong JP, Kim SD, Kang HJ, Lee YS. Development of a Korean version of the Perceived Deficits QuestionnaireDepression for patients with major depressive disorder. Clin Psychopharmacol Neurosci 2016;14:26-32.

24. Maier W, Buller R, Philipp M, Heuser I. The Hamilton Anxiety Scale: reliability, validity and sensitivity to change in anxiety and depressive disorders. J Affect Disord 1988;14:61-68.

25. Fydrich T, Dowdall D, Chambless DL. Reliability and validity of the beck anxiety inventory. J Anxiety Disord 1992; 6:55-61.

26. Spielberger CD. State-trait anxiety inventory for adults (STAl-AD) [Internet]. APA PsycTests; 1983 [cited at 2020 Sep 15]. Available from: https://doi.org/10.1037/t06496-000.

27. Jermann F, Van der Linden M, d'Acremont M, Zermatten A. Cognitive Emotion Regulation Questionnaire (CERQ). Eur J Psychol Assess 2006;22:126-131.

28. Connor KM, Davidson JR. Development of a new resilience scale: the Connor-Davidson Resilience Scale (CD-RISC). Depress Anxiety 2003;18:76-82.

29. Klein LE, Roca RP, McArthur J, Vogelsang G, Klein GB, Kirby $\mathrm{SM}$, et al. Diagnosing dementia. Univariate and multivariate analyses of the mental status examination. J Am Geriatr Soc 
1985;33:483-488.

30. Yun GJ, Chun MH, Kim BR. The effects of transcranial directcurrent stimulation on cognition in stroke patients. J Stroke 2015:17:354-358.

31. Kalu UG, Sexton CE, Loo CK, Ebmeier KP. Transcranial direct current stimulation in the treatment of major depression: a meta-analysis. Psychol Med 2012;42:1791-1800.

32. Loo CK, Alonzo A, Martin D, Mitchell PB, Galvez V, Sachdev P. Transcranial direct current stimulation for depression: 3-week, randomised, sham-controlled trial. Br J Psychiatry 2012;200: 52-59.

33. Blumberger DM, Tran LC, Fitzgerald PB, Hoy KE, Daskalakis Z). A randomized double-blind sham-controlled study of transcranial direct current stimulation for treatment-resistant major depression. Front Psychiatry 2012;3:74.

34. Loo CK, Husain MM, McDonald WM, Aaronson S, O’Reardon JP, Alonzo A, et al. International randomized-controlled trial of transcranial Direct Current Stimulation in depression. Brain Stimul 2018;11:125-133.

35. Bolognini N, Spandri V, Ferraro F, Salmaggi A, Molinari AC, Fregni $\mathrm{F}$, et al. Immediate and sustained effects of 5-day trans- cranial direct current stimulation of the motor cortex in phantom limb pain. J Pain 2015;16:657-665.

36. Meron D, Hedger N, Garner M, Baldwin DS. Transcranial direct current stimulation (tDCS) in the treatment of depression: systematic review and meta-analysis of efficacy and tolerability. Neurosci Biobehav Rev 2015;57:46-62.

37. Horvath JC, Forte JD, Carter O. Quantitative review finds no evidence of cognitive effects in healthy populations from single-session transcranial direct current stimulation (tDCS). Brain Stimul 2015;8:535-550.

38. Martin DM, Moffa A, Nikolin S, Bennabi D, Brunoni AR, Flannery W, et al. Cognitive effects of transcranial direct current stimulation treatment in patients with major depressive disorder: an individual patient data meta-analysis of randomised, sham-controlled trials. Neurosci Biobehav Rev 2018; 90:137-145.

39. D’Urso G, Dell'Osso B, Rossi R, Brunoni AR, Bortolomasi M, Ferrucci R, et al. Clinical predictors of acute response to transcranial direct current stimulation (tDCS) in major depression. J Affect Disord 2017:219:25-30. 